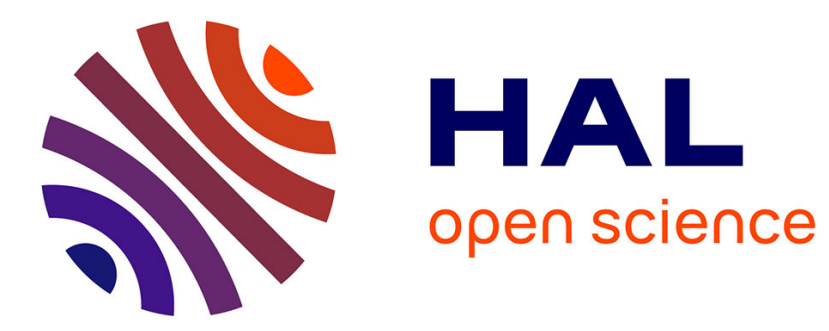

\title{
Electro-thermal simulation based on coupled Boltzmann transport equations for electrons and phonons
}

\author{
T. Nghiem, Jérôme Saint-Martin, P. Dollfus
}

\section{To cite this version:}

T. Nghiem, Jérôme Saint-Martin, P. Dollfus. Electro-thermal simulation based on coupled Boltzmann transport equations for electrons and phonons. Journal of Computational Electronics, 2016, 15 (1), pp.3 - 15. 10.1007/s10825-015-0773-2 . hal-01906694

\section{HAL Id: hal-01906694 \\ https://hal.science/hal-01906694}

Submitted on 17 Jul 2019

HAL is a multi-disciplinary open access archive for the deposit and dissemination of scientific research documents, whether they are published or not. The documents may come from teaching and research institutions in France or abroad, or from public or private research centers.
L'archive ouverte pluridisciplinaire HAL, est destinée au dépôt et à la diffusion de documents scientifiques de niveau recherche, publiés ou non, émanant des établissements d'enseignement et de recherche français ou étrangers, des laboratoires publics ou privés. 


\title{
Electro-thermal simulation based on coupled Boltzmann transport equations for electrons and phonons
}

\author{
T.T. Trang Nghiêm ${ }^{1,2}$, J. Saint-Martin ${ }^{1}$ and P. Dollfus ${ }^{1}$ \\ ${ }^{1}$ Institute of Fundamental Electronics, UMR 8622, CNRS-University of Paris-Sud, \\ Orsay, France \\ ${ }^{2}$ The center for Thermal Sciences of Lyon, UMR 5008, CNRS-INSA-University of \\ Lyon 1, Villeurbanne, France \\ Phone: +33169157283 \\ Fax: +33169154020 \\ E-mail: jerome.saint-martin@u-psud.fr
}

\begin{abstract}
To study the thermal effect in nano-transistors, a simulator based on the self-consistent solution of the Boltzmann transport equations (BTEs) for both electrons and phonons has been developed. It has been used here to investigate the self-heating effect in a 20 -nm long double gate MOSFET. In this model, a Monte Carlo (MC) solver for electrons has been coupled with a direct solver for the phonon transport. This method is particularly efficient to provide a deep insight on the out-of-equilibrium thermal dissipation occurring at the manometer scale when the length of the devices is smaller than the mean free path of both charge and thermal carriers. This approach allows us to evaluate accurately the phonon emission and absorption spectra in both real and energy spaces.
\end{abstract}

Keywords: phonon, electron, Boltzmann, Transport Equation, Silicon, MOS, DGMOS, transistors, self-heating.

\section{Introduction}

Heat conduction/dissipation and self-heating effects are taking an increasing place in the design of solid-state devices and circuits. At the simulation level, different methods have now reached a high degree of maturity to describe accurately the electronic transport under uniform lattice temperature. They include in particular the Monte Carlo (MC) method to solve the Boltzmann or Wigner transport equation and the non-equilibrium Green's function (NEGF) formalism. With different levels of approximation, these techniques are now able all derails of the material band structure [1] [2] [3], multisubband transport [4] [5] [6], quantum transport [7] [8] and scattering effects [9] [10] [11] [12]. However, introducing accurate description 
of heat conduction and its coupling with charge transport in device simulators able to consider devices of realistic size is still an issue, in spite of recent efforts.

In crystalline materials heat is mainly carried by phonons, the pseudo-particle associated with the lattice vibrations. The phonon mean free path (MFP) is usually limited by interaction with other phonons, impurities and interfaces [13]. In silicon, the mean value is estimated to be typically $300 \mathrm{~nm}$ at room temperature [14]. On distance scales larger than the MFP, the phonon system remains close to thermal equilibrium and may be well described by the classical Fourier heat equation.

However, in modern electronic devices the length of the active region is in the order of a few tens of nanometers and in the presence of a perturbation, phonon scattering events are too rare for the system to recover local thermodynamic equilibrium. Thus, in such devices the use of a macroscopic description of thermal transport as the Fourier heat equation is questionable. In this case, the phonon Boltzmann transport equation (pBTE), which has the ability to correctly describe both equilibrium and non-equilibrium phenomena, is much more relevant. Simplified equations have been derived from the pBTE and different methods for solving them have been developed with different levels of approximation. It is worth mentioning the phonon radiative transfer equation, the ballistic-diffusive equation $[15,16]$, the discrete ordinate method (DOM) [17, 18] and the lattice Boltzmann method [1921]. However, to accurately solve the pBTE, the stochastic Monte Carlo approach has been shown to be a powerful technique that can manage the details of the collision processes at the microscopic scale [13, 22-26] [27]. Additionally, it can be used in complex geometry devices. To solve the coupled electron and phonon transport equations on an equal footing within the Boltzmann approach suitable for full device simulation, it is very tempting to connect a thermal solver with an electron MC (eMC) code and to introduce a local dependence of the electron transport (scattering rates) on the state of phonon system. Different methods have been developed. In a simple approach, the eMC simulation has been coupled with a solver of the Fourier heat equation to study the size effect on the thermoelectric properties of III-V heterostructures [28]. Then a split-flux model of phonon transport has been self-consistently coupled with eMC simulation of Si FETs [29]. In 2010, Sadi anf Kelsall proposed to couple the 2D heat equation with 2D eMC simulation to describe self-heating effects in SOI transistors thanks to the positiondependence of the temperature and thermal conductivity [30]. A similar model has 
been developed to analyse the thermal effects in quantum cascade lasers [31]. To make it possible to capture out-of-equilibrium thermal phenomena, the Vasileska and Goodnick group solved the energy balance equations of thermal transport. By coupling this approach with eMC simulation, it has been possible to describe the optical phonon bottleneck in ultra-short transistors and the resulting current degradation through an analytic formulation of thermal conductivity in thin Si films [32] [33]. Kamakura et al. have implemented a MC method to solve the BTE for both electrons and phonons for 1D Si diodes with simplified phonon scattering rates but this approach has not been extended to transistors yet [34]. Recently, Ni et al. have used the phonon generation spectrum extracted from eMC simulation as input for a pBTE solver with anisotropic relaxation times and Brillouin zone to evaluate the hotspot temperature in a MOSFET [35]. This detailed description requires large computational resources [36].

In this paper, we introduce a computationally efficient approach to solving deterministically the steady-state 1D pBTE within the relaxation time approximation (RTA). This phonon transport solver has been self-consistently coupled to an eMC device simulation to study the electro-thermal effects in nanoelectronic devices. This model provides deep insight into the out-ofequilibrium phonon effects in small devices.

This paper is organized as follows. Sections 2 and 3 are dedicated to the presentation of the main features of the thermal simulator based on the direct solution of the pBTE, and on the phonon scattering mechanisms included in the model for silicon, respectively. In Section 4 this model is used to explore the different regimes of phonon transport in silicon bars of different lengths, from diffusive to ballistic limits. Next, in Section 5 we explain how this thermal transport model has been coupled to an electron Monte Carlo simulation code to build an electro-thermal device simulator. This simulator has been used to investigate the self-heating effects in an ultra-short Double Gate MOS field effect transistor (DGMOSFET). The results presented in section 6 put forward the effect of out-ofequilibrium phonon distribution resulting from high phonon generation rate at the drain-end of the channel. 


\section{Thermal simulator}

\subsection{Phonon dispersion}

The phonon dispersion in silicon is composed of six phonon modes, i.e. two transverse acoustic (TA), one longitudinal acoustic (LA) acoustic, two transverse optical (TO) and one longitudinal optical (LO) modes. In this work, the TA modes on one side and the TO modes on the other side have been considered to be degenerate, which is exact along the main crystallographic directions. For each mode $s$ the dispersion relation has been assumed to follow an analytic and quadratic expression of the form

$$
\omega_{s}(q)=\omega_{0, s}+v_{g, s} q+a_{s} q^{2}
$$

where $q$ is the modulus of the phonon wave vector. The parameters $\omega_{0, s}, v_{g, s}$ and $a_{s}$ are taken from Ref. [37] where they have been optimized to fit the actual dispersions along the direction [100]. Accordingly, the Brillouin zone was assumed to be isotropic in this model, i.e with a spherical symmetry. The maximum value of the wave vector norm $q_{\max }=\pi / a$ is the radius of the Brillouin zone where $a$ is the lattice parameter.

\subsection{Thermal transport equation}

Since the out-of-equilibrium character of phonon transport may be significant in nano-devices, the use of the Boltzmann transport formalism to study the heat diffusion is particularly relevant. In contrast to the case of charged particles, the trajectories of phonons are not modified by any external driving forces. Hence, the corresponding drift term is absent in the Boltzmann transport equation for phonons (pBTE), the steady-state form of which can be written for each mode $s$ as

$$
\vec{v}_{g, \mathrm{~s}}(\vec{q}) \cdot \vec{\nabla}_{\vec{r}} \cdot N_{\mathrm{s}}(\vec{r}, \vec{q})=-\left.\frac{\partial N}{\partial t}\right|_{\text {coll }}+G_{s}(\vec{r}, \vec{q}),
$$

where $\vec{q}$ is the phonon wave vector, $\vec{r}$ is the position vector, $\vec{v}_{g, s}$ is the group velocity, $N_{s}(\vec{r}, \vec{q})$ is the phonon distribution (i.e. the number of phonons with a wave vector $\vec{q}$ in the range $\vec{q} \mp d \vec{q} / 2),\left.\frac{\partial N}{\partial t}\right|_{\text {coll }}$ is the scattering term and $G_{s}(\vec{r}, \vec{q})$ is the generation term. 
In contrast to that of electrons, the distribution of phonons, which are bosons, can be higher than one and the equilibrium phonon distribution $N_{s}^{0}(T(\vec{r}), \vec{q})$ at a temperature $T(\vec{r})$ follows the Bose-Einstein statistics

$$
N_{s}^{0}(T(\vec{r}), \vec{q})=\frac{1}{\exp \left(\frac{\hbar \omega_{s}(\vec{q})}{k_{B} T(\vec{r})}\right)} .
$$

where $\hbar$ and $k_{B}$ are the Planck and Boltzmann constants, respectively.

\subsection{Heat generation}

The generation term $G_{s}(\vec{r}, \vec{q})$ in Eq. 2 derives from either an internal source, i.e. from the phonon bath itself, or from an external one.

The internal source only affects the acoustic modes. Its origin is the anharmonic decay of optical phonons in excess into acoustic phonons of lower energy. This phenomenon gives rise to a significant energy transfer from optical to acoustic modes and has to be taken into account to ensure the energy conservation of the system.

The external heat generation is related to the energy exchange between phonons and electrons. A difference between the phonon and electron temperatures induces a net increase of the emitted (or absorbed) phonons. In the self-consistent electrothermal loop of our model, these specific generation terms are extracted from the previous solution of electronic transport. More details about these generation terms can be found in Ref. [38], in particular about the computation of the anharmonic decay.

\subsection{Scattering term}

The scattering term has been implemented within the relaxation time approximation (RTA), i.e. in the form

$$
\frac{\partial N}{\partial t_{\text {coll }}}=-\frac{N_{\mathrm{s}}(\vec{r}, \vec{q})-N_{\mathrm{s}}^{0}\left(T_{\text {Fourier }}(\vec{r}), \vec{q}\right)}{\tau_{s}\left(T_{\text {Fourier }}(\vec{r}), \vec{q}\right)},
$$

where $\tau_{s}$ refers to the total relaxation time at the temperature $T_{\text {Fourier }}$. This approach of the scattering term provides a tractable linear expression. Its main issue is the determination of $T_{\text {Fourier }}$ for an appropriate description of the temperaturedependence of the relaxation time. This temperature is basically an unknown 
variable that could be rigorously quantified via a self-consistent solution. However to simplify the iterative process, in our model this temperature is directly estimated from a preliminary solution of a simple 1D Fourier heat equation, i.e.

$$
\frac{\partial T_{\text {Fourier }}}{\partial t}=\nabla \cdot\left(\kappa_{T} \nabla T_{\text {Fourier }}\right) \text {, }
$$

where $\kappa_{T}$ is the thermal conductivity that may depend on temperature, as will be discussed below. The input parameter $T_{\text {Fourier }}$, which is the solution of Eq. (5), is inserted in the scattering term (Eq. 4) and then Eq. (2) can be solved. The boundary conditions used to solve Eq. (5) and Eq. (2) are the same and as described below.

\subsection{Boundary conditions}

We consider the thermal transport to one-dimensional (1D). It means that for 2D or $3 \mathrm{D}$ devices the spatial phonon distribution is assumed to be uniform and the thermal fluxes to be zero along the transverse direction(s) perpendicular to the transport. It is relevant in the case of quasi-infinite transverse dimensions or in the presence of quasi thermal insulator such as $\mathrm{SiO}_{2}$ surrounding the active region, like in FD-SOI transistors. Along the transport direction, the two thermal contacts are assumed to be in equilibrium with two ideal reservoirs at temperatures $T_{1}$ and $T_{2}$, respectively. Thus, the phonon distributions of the entering phonons follow the corresponding Bose-Einstein distribution. Phonons hitting the contact interfaces are free to leave the device and their related energy disappear [19].

\subsection{Discretization scheme}

According to the previous parts, the unknown phonon distribution $N(z, \vec{q})$ in Eq. (2) depends on one real-space coordinate and three reciprocal-space coordinates, and the Boltzmann equation for each mode $s$ (LA,TA, LO and TO) may be rewritten in the form

$$
\begin{aligned}
\left(\tau_{s}\left(T_{\text {Fourier }}(z), q\right) \cdot v_{g, s}^{z}(\vec{q}) \cdot \frac{\partial}{\partial z}+1\right) N_{s}(z, \vec{q}) & =N_{s, T_{\text {Fourier }}}(z, \vec{q}) \\
& +G(z, \vec{q}) \cdot \tau_{s}\left(T_{\text {Fourier }}(z), q\right)
\end{aligned}
$$

where $v_{g, s}^{z}$ is the projection of the group velocity along the $z$ axis, the transport direction. Due to the isotropic character of the scattering terms used in this work 
(see next section), the solution of Eq. (6) remains straightforward since it can be decoupled along the directions of the reciprocal space that are independent.

The real space is discretized with a non-uniform grid and the maximum mesh size $(\Delta z)$ must be smaller than the MFP. In the reciprocal space, a Cartesian grid is applied along the three directions with wave vector components $q_{x}, q_{y}$ and $q_{z}$. The number of $q$-steps $N_{q}=q_{\max } / \Delta q$ is fixed to 100 , which ensures an accuracy of the order of one percent in the computation of the conductivity. Hence, the Brillouin zone, assumed to be spherical, is divided into about $5 \times 10^{5}$ cubic cells.

The derivative operator is approximated within the finite difference method, using either the forward $\left(\frac{\partial N_{s}^{i}}{\partial z_{i}}=\frac{N_{s}^{i+1}-N_{s}^{i}}{\Delta z_{i}}\right)$ or backward $\left(\frac{\partial N_{s}^{i}}{\partial z_{i}}=\frac{N_{s}^{i}-N_{s}^{i-1}}{\Delta z_{i}}\right)$ Euler's approximation depending on whether $v_{g, s}^{z}$ is positive or negative, respectively. By fixing the temperature at the two contacts, the final linear matrix equation is solved using a standard LU decomposition.

\subsection{Effective temperature}

In the context of non-equilibrium phonon distributions which is often encountered at the nanoscale, the concept of (standard) temperature is meaningless and considering a "phonon distribution" field is more relevant. Nevertheless, the use of an "equivalent temperature" field, called effective temperature $T_{\text {eff, }}$, and defined from the total energy of the local phonon bath resulting from the actual phonon distribution (which may be out-of-equilibrium) naturally extends the common temperature concept [39]. According to this definition, an effective temperature for each mode can be defined. It should be noted that, in contrast to what is observed in out-of-equilibrium conditions, once the equilibrium is recovered the temperature of the four modes is equal to $T_{\text {eff }}$ and corresponds to the standard temperature $T$.

\section{Scattering and thermal conductivity}

In the case of low temperature gradient within the RTA and assuming the isotropic dispersion of Eq. 1, an analytical expression of the thermal conductivity (considering only low temperature gradients) can be derived from Eq. (2), as follows [40]: 


$$
\kappa_{s}=\frac{1}{3} \sum_{s=L A, T A} \int_{0}^{q_{\max }} \frac{\mathrm{h} \omega_{s}^{2}(q)}{k_{B} T^{2}} v_{g, s}^{2}(q) \tau_{s}(q) \frac{\exp \left(X_{s}\right)}{\left[\exp \left(X_{s}\right)-1\right]^{2}} q^{2} \frac{d q}{2 \pi^{2}},
$$

where $X_{s}=\hbar \omega_{s}(q) / k_{B} T$.

In this approach, the contribution of optical modes, that exhibit weak average group velocities with respect to acoustic modes, is neglected. The total relaxation time $\tau_{s}$ in Eq. (9) is computed according to the Mathiessen's rule i.e. by summing up the contributions of all relevant scattering mechanisms. In the case of silicon, the total relaxation time includes phonon-impurity, phonon-phonon and phonon-rough interface scattering mechanisms. The modelling of these mechanisms and the resulting thermal conductivity are presented in this section.

\subsection{Phonon-Impurity relaxation time}

The relaxation time related to the Phonon-Impurity scattering mechanism used in this work follows the well-established Holland's model which results from an analysis of thermal conductivity based on a linear dispersion and fitted scattering parameters [17]. It is given by

$$
\tau_{I}(q)^{-1}=A \times \omega^{4} .
$$

where $A=1.32 \times 10^{-45} \mathrm{~s}^{3}$ and $\omega$ is the phonon angular frequency.

\subsection{Phonon-Phonon relaxation time}

The implementation of the phonon-phonon scattering in $\mathrm{Si}$ is also based on the Holland's model [41]. In this model, in the case of longitudinal acoustic phonons only one kind of scattering is considered, while for transverse acoustic phonons, normal and Umklapp mechanisms are considered separately. The corresponding expressions of these relaxation times are reminded in Table 1.

\begin{tabular}{|l|l|}
\hline Scattering mechanisms & Relaxation Time \\
\hline TA Normal scattering & $\tau_{T A}(q, T)^{-1}=B_{T N} \times \omega(q)^{3} \times T^{4}$ \\
TA Umklapp scattering & $\tau_{T A}(q, T)^{-1}=B_{T U} \times \omega(q)^{2} / \sinh \left(\mathrm{h} \omega(q) / k_{B} T\right)$ \\
LA scattering & $\tau_{L A}(q, T)^{-1}=B_{L} \times \omega(q)^{2} \times T^{3}$ \\
Optical phonon & $\tau_{O}{ }^{-1}=3.5 \mathrm{ps}$ \\
\hline
\end{tabular}


Table 1. Expressions of relaxation times for phonon-phonon interactions in Bulk Si. The values of parameters $B_{T N}, B_{T U}$ and $B_{L}$ are discussed in the text and given in Table 2

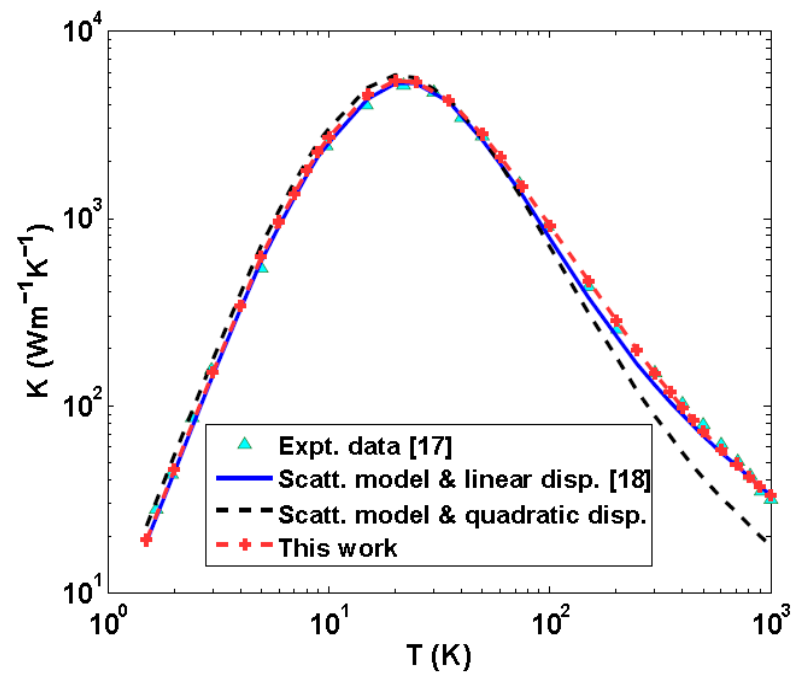

Figure 1. Thermal conductivities in bulk Si as a function of the temperature: experimental data [42] (triangles), model of Holland [41] (solid line), scattering parameters from [41] with the dispersion of Eq. 1 (dashed line) and our work (red crosses).

In Figure 1, the symbols represent experimental measurements of the thermal conductivity in bulk $\mathrm{Si}$. The conductivities related to phonon-phonon interactions in the acoustic branches and computed via Eq. 6 are represented by lines. Obviously, the original Holland's model (blue solid line) based on a linear dispersion for acoustic phonons fits the experiments. Nevertheless, the results obtained with the original parameters $B_{T N}, B_{T U}$ and $B_{L}$ (black dashed line) and a quadratic dispersion relation (Eq. 1) are disappointing, especially at high temperature.

\begin{tabular}{|c|l|l|}
\hline$B_{T N}=10.5 \times 10^{-13} \mathrm{~K}^{-3}$ & if $\omega<\omega_{\text {cut }}: B_{T U}=0$ & $B_{L}=1.18 \times 10^{-24} \mathrm{sK}^{-3}$ \\
& else $: B_{T U}=2.89 \times 10^{-18} \mathrm{~s}$ & \\
& where $\omega_{\text {cut }}=\omega\left(q_{\max } / 2\right)$ & \\
\hline
\end{tabular}

Table 2. Set of parameters used for phonon-phonon interactions in Bulk Si when considering a quadratic dispersion.

However, if these parameters are tuned to the values given in Table 2, the computed conductivities (red dashed line and red crosses) perfectly reproduce the experimental data and even better that those of the original model for a temperature ranging from $2 \mathrm{~K}$ to $1000 \mathrm{~K}$.

From 150 to $600 \mathrm{~K}$, the evolution of the experimental conductivities is proportional to $T^{\alpha}$ and is well fitted by

$$
\kappa_{T}=\frac{3.09 \times 10^{5}}{T^{1.34}}
$$


Optical phonon modes are often neglected in investigations of thermal conductivity. However, in the presence of excited optical phonon populations, their anharmonic decay into lower energy phonons is a fundamental phenomenon which governs the energy relaxation and thus the evolution of the non-equilibrium (hot) phonon populations [43]. In our electro-thermal simulation the optical phonon modes (LO and TO) can be included and a constant relaxation time is used. This time is chosen according to the measurement of Ref. [44] and is given in Table 1.

\subsection{Phonon-boundary relaxation time}

At the boundaries, phonons can undergo either a specular or a diffusive reflection. The specular reflections have no impact on the phonon conductivity, in contrast to the diffusive ones that are caused by the defects at the reflecting interfaces. These defects randomize the velocity of the involved phonons along the interface. Moreover, these boundary scatterings become predominant below $50 \mathrm{~K}$ in silicon [36] due to the "freezing out" of phonon-phonon interactions. Additionally, these interface effects are size-dependent, so that we have to consider separately the bulk and the thin film boundary scattering mechanisms.

In bulk Silicon, the Holland's model is once again considered. The related relaxation time has the form [17]

$$
\tau_{B, b u l k}^{-1}(q)=\frac{v_{g, s}(q)}{L \times F}
$$

where $L=7.16 \times 10^{-3} \mathrm{~m}$ is the equivalent sample size and $F=0.68$ is a geometric factor.

In the case of thin films where the characteristic time between two collisions at interfaces is in the same order of magnitude as the total relaxation time, the relaxation time associated with interface diffusion is expressed as [18]

$$
\tau_{B}(\stackrel{\mathrm{r}}{q})^{-1}=\min \left\{\frac{L_{i}}{v_{g, s}^{i}(\stackrel{1}{q})} \times \frac{1+p(\stackrel{\mathrm{r}}{q})}{1-p(\stackrel{1}{q})}\right\},
$$

where $p(\stackrel{\mathrm{r}}{q})=\exp \left(-4 \stackrel{\mathrm{r}}{q^{2}} \Delta^{2} \cos ^{2} \theta_{B}\right), i$ stands for the direction $x, y$ or $z, L_{i}$ is the length of the device along the direction $i$. The coefficient of specular reflections $p(\vec{q})$ varies from 0 to 1 depending on both the interface roughness parameter $\Delta$, i.e. the standard deviation of interface fluctuations, and the incident angle of the phonon $\theta_{B}$. 
In Figure 2, the evolution of the in-plane conductivity as a function the roughness is plotted for several film widths at ambient temperature. While the roughness parameter is not relevant in the case of thick films, to validate the model it is mandatory to reproduce the experimental data for films thinner than $100 \mathrm{~nm}$.

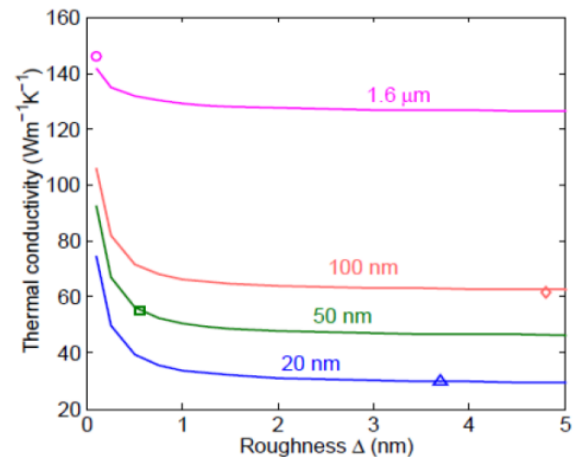

Figure 2. Thermal conductivity as a function of the roughness parameter $\Delta$ for 20, 50, $100 \mathrm{~nm}$ and $1.6 \mu \mathrm{m}$ film thickness. Symbols are the experimental data for $T_{S i}=20 \mathrm{~nm} \quad$ (triangle)[45], $50 \mathrm{~nm}$ (square)[45], $100 \mathrm{~nm}$ (diamond)[46] and $1.6 \mu \mathrm{m}$ (circle) [47].

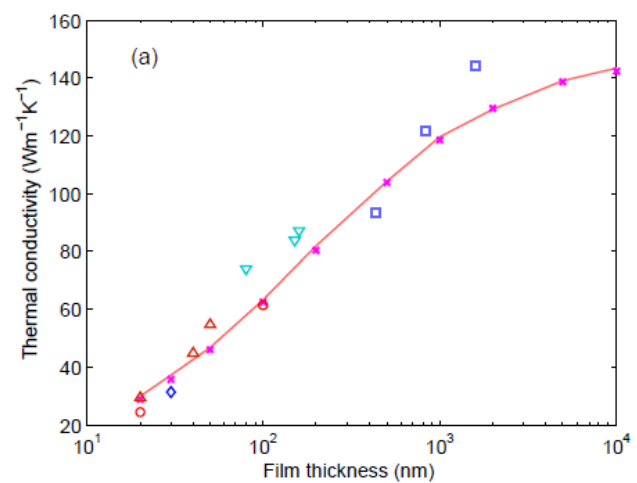

Figure 3. In-plane Thermal conductivities in silicon thin-films at $300 \mathrm{~K}$ as a function of the film width. Continuous lines: our analytical RTA model, symbols: experimental data [47] (squares), [14] (blue triangle),[45] (red triangles), [46] (red circles), [48] (blue quadrangle).

However, as shown in Figure 2, the value of the roughness parameter needed to reproduce the experimental conductivity is relatively high and it is reasonable to assume fully diffusive interfaces, i.e. $p(\vec{q})=0$. In an "in-plane" model for films with a Silicon thickness $T_{S i}$ and a transverse group velocity $v_{g, s}^{T}$, the simple form of the phonon-boundary relaxation time is

$$
\tau_{B, f i l m}^{-1}=v_{g, S}^{T}(\vec{q}) / T_{S i}
$$

As illustrated in Figure 3, this simple model matches accurately the experimental conductivities for film thicknesses ranging from $20 \mathrm{~nm}$ to $10 \mu \mathrm{m}$.

\section{From diffusive to ballistic heat transport}

This section is dedicated to the study of heat transport regimes. The pBTE (Eq. 2) was numerically solved as described in section 2 , including the relaxation times of section 3. Several temperature gradients were applied on Silicon bars of different lengths. Only the acoustic (LA and TA) modes were considered as the average group velocities of optical modes are weak compared to acoustic ones. 


\subsection{Temperature profiles and non-linear effects}

Temperature profiles in a $5 \mu \mathrm{m}$-long $\mathrm{Si}$ bar under a high temperature difference $\left(T_{1}\right.$ $=500 \mathrm{~K}$ and $T_{2}=250 \mathrm{~K}$ ) are displayed in Figure 4.

In this device much longer than the MFP, the heat transport is diffusive. However, the evolution of the temperature along the bar is obviously non-linear. Actually, for such a high temperature gradient, this non-linear behaviour is due to the temperature-dependence of the thermal conductivity, described by Eq. 7. It is worth noting that in this case our numerical pBTE results are in accordance with an analytical solution of the diffusive Fourier heat equation (derived in the Appendix). In the case of smaller temperature gradient $\left(T_{1}=310 \mathrm{~K}\right.$ and $\left.T_{2}=290 \mathrm{~K}\right)$, the temperature profiles for several bar lengths are plotted in Figure 5. In a long bar where the transport is diffusive; the profile is linear in accordance with the Fourier's law with a constant conductivity. In contrast, in very short bars much smaller than the MFP, the transport tends to its ballistic limit and thus the temperature profile tends to a step function. The temperature in the ultra-short device is very close to the ballistic temperature limit $T_{B a l}=\left(T_{1}^{4} / 2+T_{2}^{4} / 2\right)^{1 / 4}=300.5 \mathrm{~K}$ derived from the Stefan-Boltzmann law [49].

The temperature evolution between these two limit cases shows a smooth change from a quasi-diffusive transport regime occurring in the $200 \mathrm{~nm}$-long bar and a quasi-ballistic regime in the $20 \mathrm{~nm}$ one.

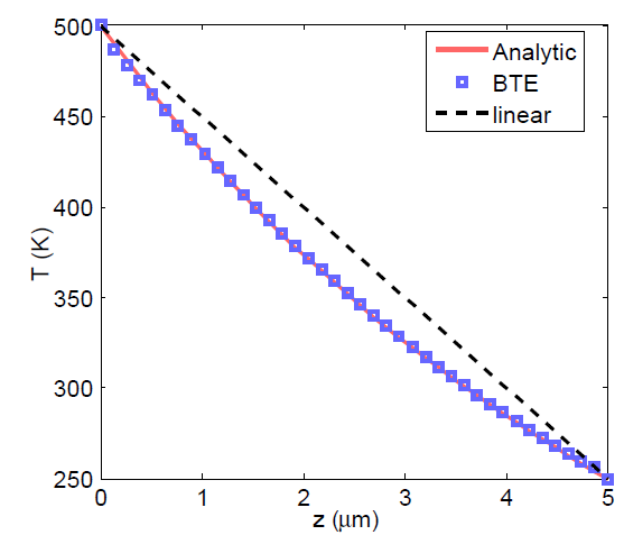

Figure 4. Temperature profiles in a $5 \mu \mathrm{m}$ long Si bar: our numerical pBTE (symbols) and analytical calculation (solid line) for a large temperature gradient.

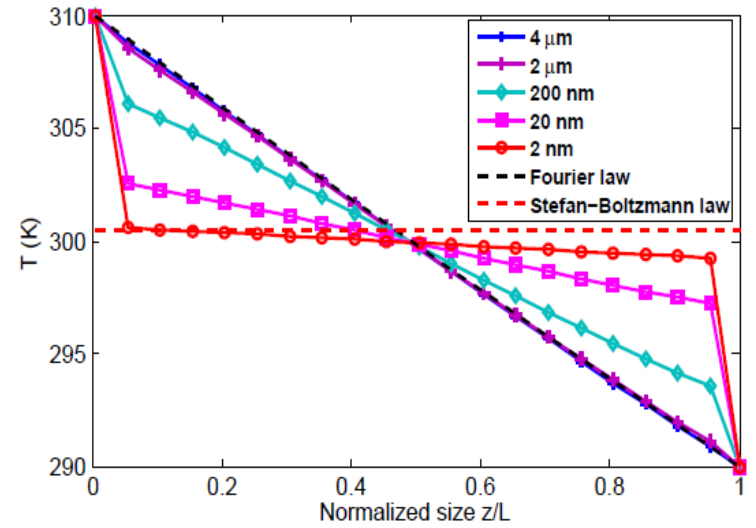

Figure 5. Effective temperature profiles for different Si bar lengths L from 4 um down to $2 \mathrm{~nm}$ for a small temperature gradient. 


\subsection{From diffusive to ballistic phonon distributions}

To further investigate the transition between the diffusive and ballistic regimes the phonon distributions in $\vec{q}$-space have been extracted. These spectra are very specific to our approach based on $\mathrm{pBTE}$ that provides microscopic insights into the phonon transport in all transport regimes.

The evolution of TA (for LA see Ref. [50]) phonon distributions from the hot contact at $T_{h}=400 \mathrm{~K}$ to the cold contact at $T_{c}=300 \mathrm{~K}$ is displayed in Figure 6 for bar lengths of $2 \mu \mathrm{m}, 200$ and $2 \mathrm{~nm}$ which correspond to diffusive, intermediate and ballistic regimes, respectively. The distributions are computed as a function of the component $q_{z}$, of the wave vector.

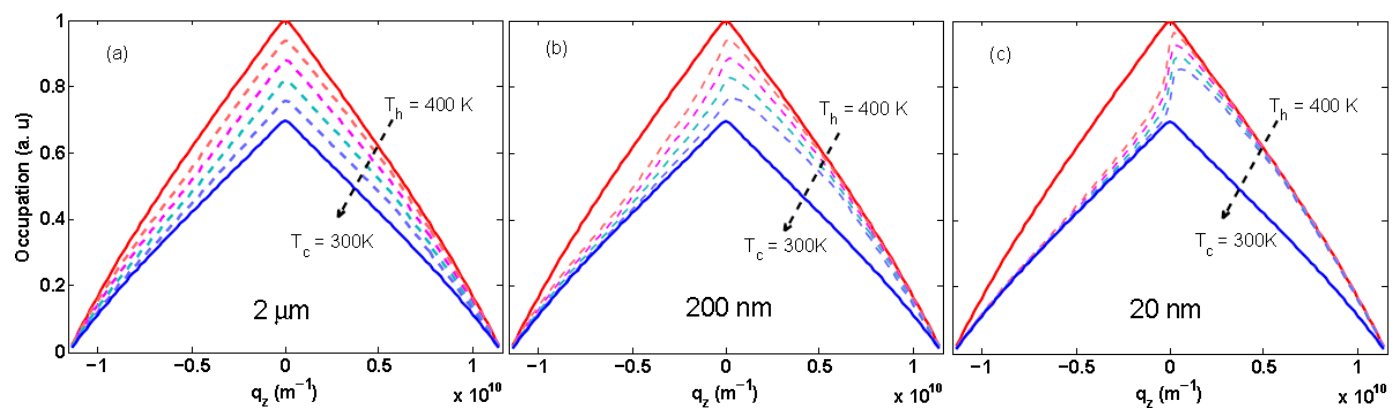

Figure 6. Evolutions of the longitudinal wave vector $q_{z}$ spectra for TA phonons at different positions regularly distributed between the two contacts for (a) $2 \mu \mathrm{m}$ (b) $200 \mathrm{~nm}$ and (c) $20 \mathrm{~nm}$ bar lengths. The arrows show the direction from contact 1 to contact 2.

Obviously, the phonon occupations depend on temperature and decrease from hot to cold contacts. Besides, in all samples, the applied boundary conditions impose the phonon distributions at these two contacts to be given by the corresponding Bose-Einstein occupation function.

For a $2 \mu \mathrm{m}$-long sample which is longer than the MFP, the transport is quasidiffusive. The distributions are quasi-symmetric and their shape is a clear manifestation of the local thermodynamic quasi-equilibrium all along the device.

In contrast, the evolution of phonon occupations in the $20 \mathrm{~nm}$ long bar are strongly dissymmetric, except at the contacts. The shape of the distributions inside the device strongly differs from those at equilibrium and is typical of ballistic transport [51] [52]. In this ultra-short sample the phonons travel without experiencing any scattering event. Hence, the distribution of phonons remains unchanged during their crossing of the device and is exactly the same as that injected from the emitting contact. Therefore, the phonons may be separated in two distinct populations: the negative and the positive parts of the distribution. The negative (positive) part, i.e. 
with $q_{z}<0\left(q_{z}>0\right)$, is related to phonons flowing from contact 2 (1) to contact 1 (2).

An intermediate regime in which the influence of scattering is neither dominant nor negligible is observed in the $200 \mathrm{~nm}$-long bar. The occupations are also asymmetric and two populations can be distinguished. However, for positive (negative) $q_{z}$ the transition from contact 1 (2) to that of contact 2 (1) is not as abrupt as in the ballistic case. It should be highlighted here that a strong advantage of our numerical approach for heat transport is its capability to capture accurately the physical features of such an intermediate transport regime.

\section{Electro-thermal Simulator}

To investigate self-heating effects in electronic devices, the thermal solver presented in the previous part has been coupled to an electron transport simulator. The resulting electro-thermal simulator is described in this section.

\subsection{Electron Monte Carlo simulation}

To solve the electron transport equation we have used a homemade Ensemble Monte Carlo simulation for electrons (eMC). In this approach the Boltzmann equation is solved using a stochastic calculation of particle trajectories selfconsistently coupled with the Poisson equation. In this version of the code the conduction band of electrons in silicon is described through an analytical nonparabolic model for the six ellipsoidal $\Delta$ valleys. This model provides a density of states (DOS) close to that of a full-band description below about $1.5 \mathrm{eV}$ [53]. Degeneracy effects and quantum corrections [4] are not included here. All details of the band structure and the scattering parameters used for acoustic phonon, inter valley phonon, ionized impurity and oxide interface roughness scattering mechanisms can be found in Ref. [54]. This simulator reproduces well the experimental mobility in unstrained (and strained) bulk-Si [54] and allows the detailed investigation of non-stationary effects [51].

\subsection{Heat generation and phonon dispersion}

The cartography of the phonon temperature is an input to the eMC simulation that provides the heat generation term needed in Eq. 2 via the local counting of electronphonon scattering events. In contrast to macroscopic approaches assuming local 
equilibrium, this method includes an accurate description of the energy transfers between phonons and hot electrons and gives access to their accurate location [38]. As in most common eMC models, the phonon dispersions used to conveniently compute the intervalley scattering rates are assumed to be wave vector-independent. Fortunately, the simulator provides information about the wave vector of the phonon selected to be involved in the electron-phonon scattering event: $\vec{q}=\overrightarrow{k^{\prime}}-\vec{k}$ where $\vec{k}$ and $\overrightarrow{k^{\prime}}$ are the electron wave vectors in the initial and final states, respectively. If the wave vector $\vec{q}$ is out of the first actual Si Brillouin zone which is tetrahedron, a relevant lattice vector is added to manage only wave vectors in the first Brillouin zone. Then, the angular frequency of an interacting phonon is computed afterward from the wave vector $\vec{q}$ and the quadratic dispersion of Eq. 1 . For both acoustic and optical phonons, we have checked that this approach does not break the average energy conservation between electrons and phonons (see more details in Ref. $[38,50])$.

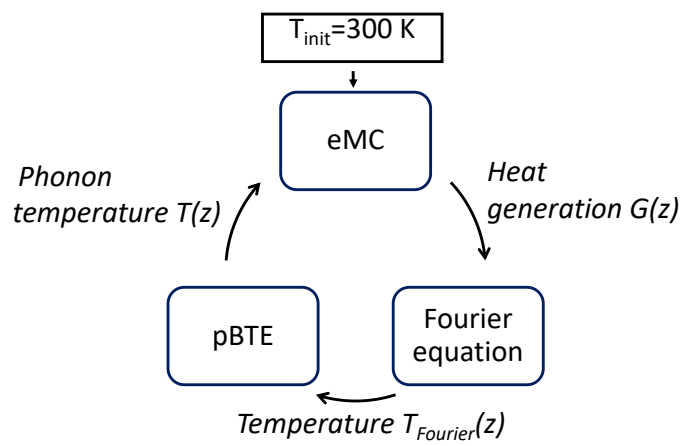

Figure 7. Main steps of the coupled electro-thermal simulator.

\subsection{Self-consistent alogorithm}

The schematized coupling procedure between electron and phonon transport is illustrated in Figure 7. The coupled simulation starts with an isothermal (300 K) eMC simulation (referred as "open loop" simulation). Then, the net phonon generation rates, which are functions of both the position and the phonon frequency, are extracted from eMC outputs and used as inputs for both the Fourier heat equation and pBTE solver described in Section 2. The resulting local effective temperature is then re-injected in the eMC simulator. It should be mentioned that only the effective temperature fields are exchanged between steps because in standard situations the phonon occupations do not significantly differ from their equilibrium distribution [38]. Next, all electron scattering rates, as electron-phonon 
and electron-impurity scattering rates, are re-calculated in each cell according to the position-dependent $T_{\text {eff. }}$. Then, the eMC simulation is performed again with this new field of temperature. This three-step process, that successively includes an electron and a phonon transport simulation, is called a loop. The convergence is reached when the effective temperature field between two consecutive loops is smaller than the target value. It should be noted that in most cases the convergence is reached after only three loops.

\section{Self-heating effects in DG-MOS}

The electro-thermal simulator presented in Section 4 was used to investigate the self-heating effect in a $20 \mathrm{~nm}$ long DGMOS transistor. As presented later, hot electrons play a significant role in this device; thus the optical modes and the related LTO decay are included in the pBTE solving.

A 2D cross-section of the studied Si DG-MOSFET is schematized in Figure 8. The device consists of three regions: the highly $\mathrm{N}$-doped $\left(5 \times 10^{19} \mathrm{~cm}^{-3}\right)$ source and drain regions and the $20 \mathrm{~nm}$ long and non-intentionally doped $\left(10^{15} \mathrm{~cm}^{-3}\right)$ channel. The source length is $50 \mathrm{~nm}$, while the drain length is extended to $150 \mathrm{~nm}$ to make it larger than the relaxation length of hot electrons. The thickness of the Si-film is $20 \mathrm{~nm}$.

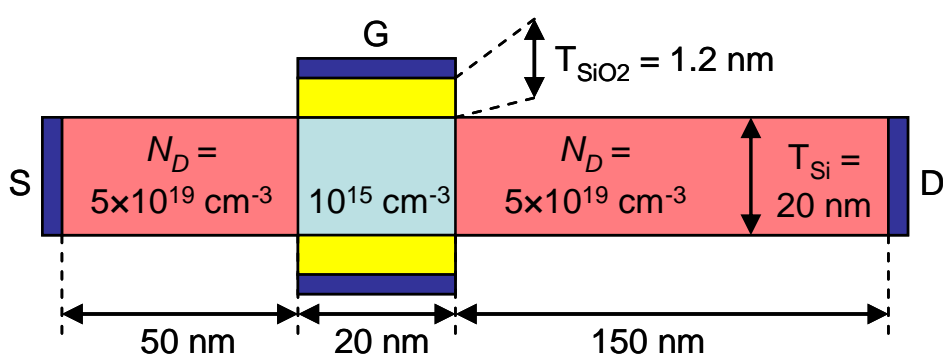

Figure 8. Schematic cross-section of the simulated device.

\subsection{Heat generation}

The profile of heat power density resulting from the initial isothermal (300 K) eMC simulation (the 'open loop') is plotted in Figure 9.a. This MC result (solid line) indicates that hot electrons heated in the channel, that is the high electric field region, transfer their energy to the phonon bath mainly in the drain region, and for a significant part of them far into the drain extension. This result considerably 
diverges from the prediction based on local equilibrium (dashed line) which estimates the heat power as the product of the local electric field $(\vec{E})$ and the local current density $(\vec{J})$. This macroscopic approach, neglecting the important out of equilibrium effects occurring in this DGMOS, would predict a maximum of dissipated heat inside the channel where the field is high.

Additionally, the generated heat power is positive all along the device, i.e. no thermoelectric cooling is observed even near the source/channel potential barrier where the electric field is negative.

In Figure 9.b, the phonon generation rates computed for each phonon mode are plotted along the source-drain direction. In the source region where electrons remain close to equilibrium (cf. [38]) the emitted phonons belong mainly to the LA and TO branches while the other modes have no noticeable effect. In the channel, where the transit time of electrons is very short compared to the scattering times, the net phonon generation is almost zero for all modes. In the drain, where the phonon generation rate is clearly the strongest, the main emission processes are once again due the LA and TO modes. However a significant contribution of TA phonons can be observed near the channel, with a shorter decay length. From the channel-drain junction the decaying of the total phonon generation rate is characterized by a decay length of about $28 \mathrm{~nm}$ for $V_{d s}=1.0 \mathrm{~V}$, which is much shorter than the length of the drain extension. Thus, near the drain contact the heat generation rate of each mode reaches a local equilibrium state very similar to that in the source.
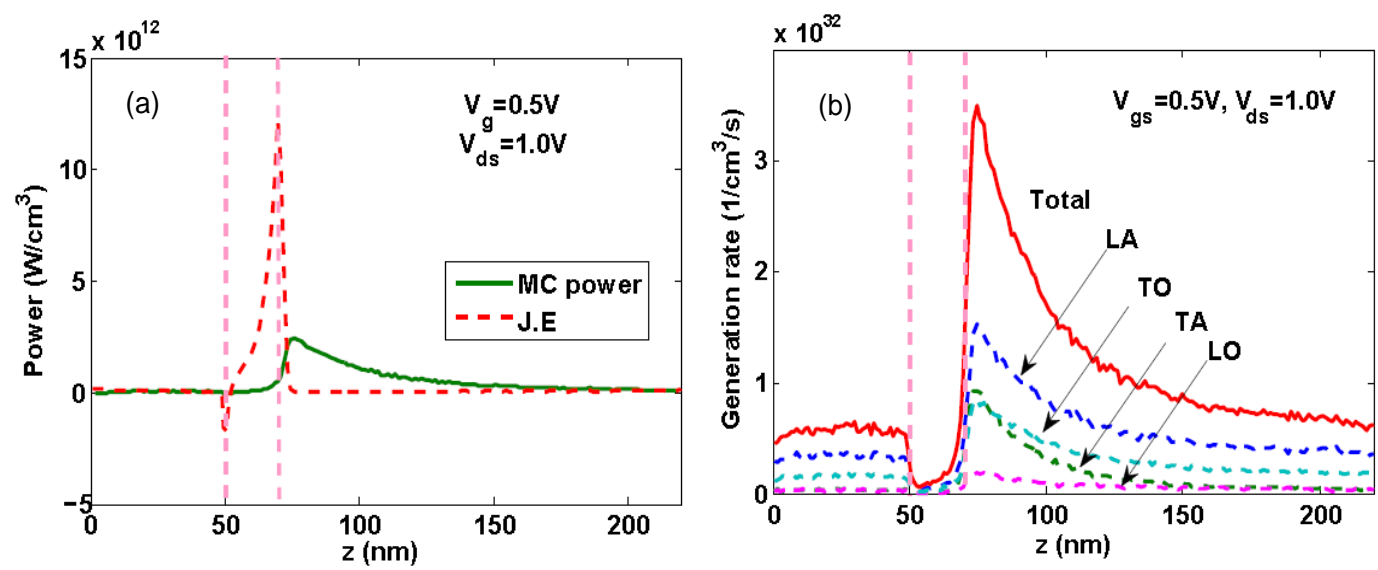

Figure 9. At $V_{g s}=0.5 \mathrm{~V}$ and $V_{d s}=1.0 \mathrm{~V}$. (a) Heat power density extracted from eMC simulation (green solid line) and from the product $\vec{J} \cdot \vec{E}$ (dashed red line). (b) Net phonon generation rate per mode (dashed lines) and total generation rate (continous line) due to electron-phonon scattering, from eMC simulation. 


\subsection{Self-heating effect}

The heat generation computed from the initial eMC simulation presented above has been included in the phonon transport equation (p-BTE) to initiate the iterative algorithm presented in Section 5.

We plot in Figure 10.a the profile of the effective temperature $T_{\text {eff }}$ at $V_{g s}=0.5 \mathrm{~V}$ and $V_{d s}=1.0 \mathrm{~V}$ after each loop. It confirms that the convergence is obtained after only three loops. The feedback resulting from the iterative process induces a slight reduction of the hotspot temperature from $441 \mathrm{~K}$ in the $1^{\text {st }}$ loop to $433 \mathrm{~K}$ after convergence. Besides, near the two contacts with the thermal reservoirs, a temperature drop can be observed. This drop is higher near the drain contact because the phonon transport is further from equilibrium than in the source side.

In Figure 10.b, we study the effect of the interface quality of the $\mathrm{Si}-\mathrm{SiO}_{2}$ interface by artificially tuning the roughness parameter $\Delta$. Switching from a quasi-specular $(\Delta=0.35 \mathrm{~nm})$ to a quasi-diffusive interface $(\Delta=3 \mathrm{~nm})$ significantly modifies the thermal resistance of the Si bar leading to an important change in the hot spot temperature. At the studied bias, the $T_{\text {eff }}$ difference can reach $50 \mathrm{~K}$ in the hot spot. More interestingly, this figure shows the difference between the temperature profiles of $T_{\text {eff }}$ resulting from the pBTE (solid lines) and of $T_{\text {Fourier }}$ given by the simple heat equation (dashed lines). This difference quantifies the influence of the out-of-equilibrium thermal effects. Near the source and drain contacts these two temperatures are similar and the temperature profile is linear as expected in nearequilibrium regime. However, they significantly differ in the vicinity of the region of strong heat generation where the $T_{\text {eff }}$ profiles exhibit a more complex evolution than the $T_{\text {Fourier }}$ ones. The $T_{\text {eff }}$ peak is more abrupt and occurs in front of the channel/drain junction. The difference between $T_{\text {Fourier }}$ and $T_{\text {eff }}$ reaches $8 \mathrm{~K}$ in the case of weak thermal resistance i.e. for $\Delta=0.35 \mathrm{~nm}$. This difference is reduced when the thermal transport is degraded $(\Delta=3 \mathrm{~nm})$. 

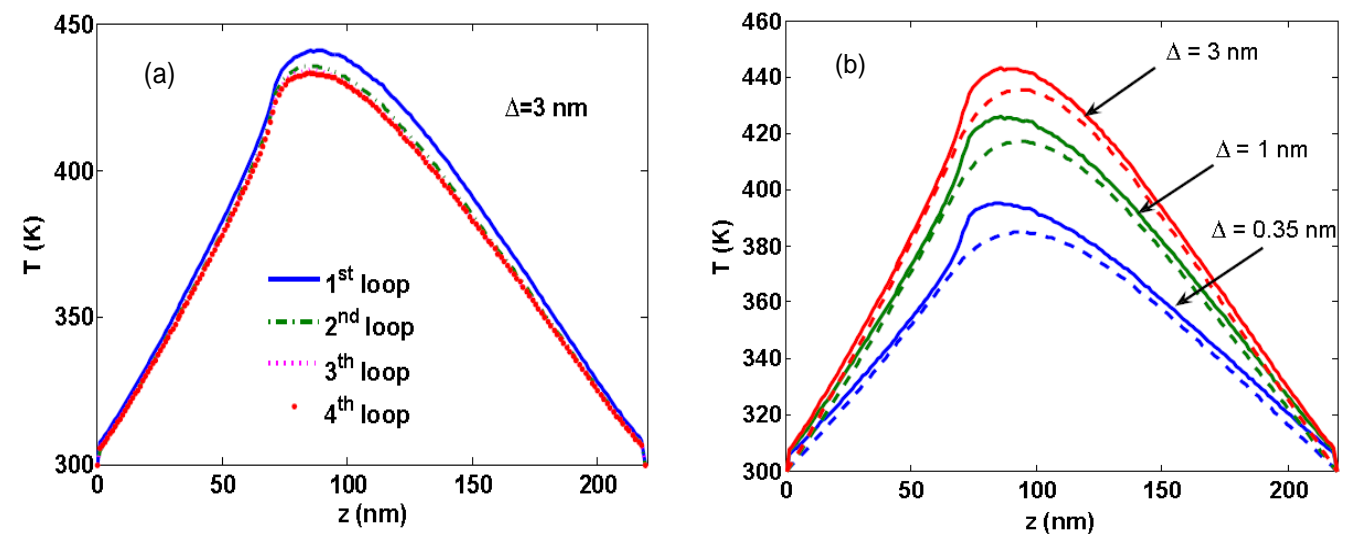

Figure 10. At $V_{g}=0.5 \mathrm{~V}$ and $V_{d s}=1.0 \mathrm{~V}$. (a) Evolution of the effective temperature $T_{\text {eff }}$ along the device for several loop numbers. (b) After convergence, profiles of $T_{\text {eff }}$ (solid lines) and $T_{\text {Fourier }}$ (dashed lines) for roughness parameters $\Delta=0.35 \mathrm{~nm}, 1 \mathrm{~nm}$ and $3 \mathrm{~nm}$.

\subsection{I-V characteristics}

The drain current characteristics $I_{d^{-}} V_{d s}$ at a gate voltage $V_{g s}=0.5 \mathrm{~V}$ and the transfer characteristics $I_{d}-V_{g s}$ at $V_{d s}=1.0 \mathrm{~V}$ are shown in Figure 11.a and b, respectively. To highlight the effect of self-heating, the isothermal open loop simulations have been compared with electro-thermal simulations. As expected, the impact of self-heating manifests itself mainly at high drain current, when the enhancement of channel temperature is sufficient to induce a significant increase of the number of scattering events in the active region. It induces a drain current degradation of $6.9 \%$ and $20 \%$ for $V_{d s}=0.5 \mathrm{~V}$ and $1.0 \mathrm{~V}$, respectively. Moreover, the maximum value of transconductance $g_{m}=d I_{d} / d V_{g s}$ is degraded too.
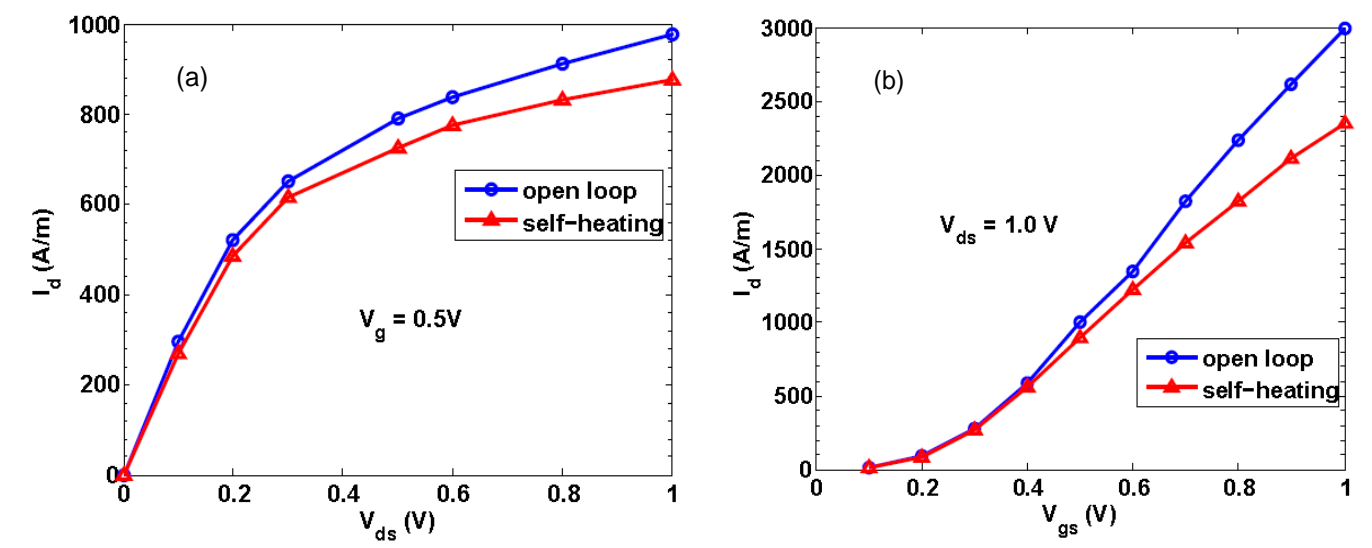

Figure 11. (a) $I_{d^{-}} V_{d s}$ at $V_{g}=0.5 \mathrm{~V}$ and (b) $I_{d^{-}} V_{g s}$ at $V_{d s}=1.0 \mathrm{~V}$. Open loop (blue lines with circular symbols) vs. electro-thermal simulations (red lines with triangular symbols). Roughness parameter $\Delta=3 \mathrm{~nm}$. 


\section{Conclusion}

We have presented a numerical method to solve the stationary Boltzmann transport equation for phonons (pBTE) in the relaxation time approximation, considering quadratic phonon dispersions. A specificity of our approach is to evaluate the equilibrium temperature to be considered in the expressions of scattering relaxation times via a preliminary solution of the Fourier heat equation. Using adjusted parameters of the Holland's model of phonon scattering, our model reproduce the thermal conductivity in silicon in the full 100-600 K temperature range. In Si films, the in-plane conductivity is also well reproduced. Besides, our advanced thermal simulator is versatile and able to describe the heat transfer in all diffusive, intermediate and ballistic transport regimes. It also provides a deep microscopic insight into the phonon behaviour such as the local phonon occupation spectra. This pBTE simulator has been coupled with our home-made Ensemble Monte Carlo simulation for electrons to study the self-heating effects in a $20 \mathrm{~nm}$-long DGMOSFET. The convergence is quickly reached, i.e. usually after only three loops. In simulations with contacts perfectly thermalized at $300 \mathrm{~K}$, the effective temperature can exceed $400 \mathrm{~K}$ in the hot spot of the transistor in on-state. Besides, it has been shown that the heat Fourier equation is not able to catch accurately the exact location and value of this temperature overshoot. The self-heating increases the detrimental effects of the access resistances and finally, the drain current can be reduced by a factor of $20 \%$ at high applied voltage.

This study could be extended in further works to investigate other devices and to extend to $2 \mathrm{D}$ configuration to take into account the interface effects.

\section{Appendix:}

\section{Diffusive equation and temperature}

\section{dependent diffusivity}

In steady-state, without heat generation, the diffusive heat equation can be reduced to

$$
\nabla\left(D_{T} \nabla T\right)=0
$$


Where $D_{T}$ is the thermal diffusivity where $D_{T}=\kappa_{T} /\left(\rho \times c_{s}\right) . \kappa_{T}$ is the thermal conductivity, $c_{s}$ the specific heat and $\rho$ the mass density.

The evolution of Silicon thermal diffusivity $D_{T}$ with respect to temperature $\mathrm{T}$ is assumed to be

$$
D_{T}=C \cdot T^{\alpha} .
$$

where $C$ and $\alpha$ are the fitting parameters.

Substituting (A-2) into (A-1) and noting that $T^{\alpha} \nabla T=\frac{\nabla T^{\alpha+1}}{\alpha+1}$, it yields a Laplace's equation for the variable $U=T^{\alpha+1}$.

By integrating the above equation in its $1 \mathrm{D}$ form with temperatures $T_{h}($ at $\mathrm{z}=0$ ) and $T_{c}($ at $\mathrm{z}=\mathrm{L})$ at the 2 boundaries, it gives:

$$
T=\left(\frac{z}{L} \cdot T_{c}^{\alpha+1}+\left(1-\frac{z}{L}\right) \cdot T_{h}^{\alpha+1}\right)^{1 /(\alpha+1)}
$$

\section{Acknowledgements}

This work was partially supported by the French ANR through project NOE (12JS03-006-01).

\section{References}

1. Fischetti, M.V. and S.E. Laux, Monte Carlo analysis of electron transport in small semiconductor devices including band-structure and space-charge effects. Physical Review B, 1988. 38(14): p. 9721.

2. Jungemann, C. and B. Meinerzhagen, Hierarchical device simulation: the Monte-Carlo perspective. 2003: Springer Science \& Business Media.

3. Luisier, M. and G. Klimeck, Atomistic full-band simulations of silicon nanowire transistors: Effects of electron-phonon scattering. Physical Review B, 2009. 80(15): p. 155430.

4. Saint-Martin, J., et al., Multi sub-band Monte Carlo simulation of an ultrathin double gate MOSFET with 2D electron gas. Semiconductor science and technology, 2006. 21(4): p. L29.

5. Lucci, L., et al., Multisubband Monte Carlo study of transport, quantization, and electron-gas degeneration in ultrathin SOI n-MOSFETs. Electron Devices, IEEE Transactions on, 2007. 54(5): p. 1156-1164.

6. Wang, J., E. Polizzi, and M. Lundstrom, A three-dimensional quantum simulation of silicon nanowire transistors with the effective-mass approximation. Journal of Applied Physics, 2004. 96(4): p. 2192-2203. 
7. Querlioz, D., et al., A study of quantum transport in end-of-roadmap DGMOSFETs using a fully self-consistent Wigner Monte Carlo approach. Nanotechnology, IEEE Transactions on, 2006. 5(6): p. 737-744.

8. Anantram, M., M.S. Lundstrom, and D.E. Nikonov, Modeling of nanoscale devices. Proceedings of the IEEE, 2008. 96(9): p. 1511-1550.

9. Esseni, D., P. Palestri, and L. Selmi, Nanoscale MOS transistors: semiclassical transport and applications. 2011: Cambridge University Press.

10. Poli, S., et al., Size dependence of surface-roughness-limited mobility in silicon-nanowire FETs. Electron Devices, IEEE Transactions on, 2008. 55(11): p. 2968-2976.

11. Cavassilas, N., et al., One-shot current conserving quantum transport modeling of phonon scattering in n-type double-gate field-effect-transistors. Applied Physics Letters, 2013. 102(1): p. 013508.

12. Niquet, Y.-M., et al., Quantum calculations of the carrier mobility: Methodology, Matthiessen's rule, and comparison with semi-classical approaches. Journal of Applied Physics, 2014. 115(5): p. 054512.

13. Mazumder, S. and A. Majumdar, Monte Carlo study of phonon transport in solid thin films including dispersion and polarization. Journal of Heat Transfer, 2001. 123(4): p. 749-759.

14. Ju, Y. and K. Goodson, Phonon scattering in silicon films with thickness of order 100 nm. Applied Physics Letters, 1999. 74(20): p. 3005-3007.

15. Cahill, D.G., et al., Nanoscale thermal transport. Journal of Applied Physics, 2003. 93(2): p. 793-818.

16. Cahill, D.G., et al., Nanoscale thermal transport. II. 2003-2012. Applied Physics Reviews, 2014. 1(1): p. 011305.

17. Volz, D.L., Jean-Bernard Saulnier, Sebastian, Clamped nanowire thermal conductivity based on phonon transport equation. Microscale Thermophysical Engineering, 2001. 5(3): p. 191-207.

18. Terris, D., et al., Prediction of the thermal conductivity anisotropy of Si nanofilms. Results of several numerical methods. International Journal of Thermal Sciences, 2009. 48(8): p. 1467-1476.

19. Sellan, D., et al., Cross-plane phonon transport in thin films. Journal of Applied Physics, 2010. 108(11): p. 113524.

20. Heino, P., Lattice-Boltzmann finite-difference model with optical phonons for nanoscale thermal conduction. Computers \& Mathematics with Applications, 2010. 59(7): p. 2351-2359.

21. Nabovati, A., D.P. Sellan, and C.H. Amon, On the lattice Boltzmann method for phonon transport. Journal of Computational Physics, 2011. 230(15): p. 5864-5876.

22. Lacroix, D., K. Joulain, and D. Lemonnier, Monte Carlo transient phonon transport in silicon and germanium at nanoscales. Physical Review B, 2005. 72(6): p. 064305.

23. Chen, Y., et al., Monte Carlo simulation of silicon nanowire thermal conductivity. Journal of Heat Transfer, 2005. 127(10): p. 1129-1137.

24. Essner, O., et al. Improved Monte Carlo algorithm of phonon transport in semiconductor nanodevices. in Journal of Physics: Conference Series. 2007. IOP Publishing.

25. Randrianalisoa, J. and D. Baillis, Monte Carlo simulation of steady-state microscale phonon heat transport. Journal of Heat Transfer, 2008. 130(7): p. 072404. 
26. Wong, B.T., M. Francoeur, and M. Pinar Mengüç, A Monte Carlo simulation for phonon transport within silicon structures at nanoscales with heat generation. International Journal of Heat and Mass Transfer, 2011. 54(9): p. 1825-1838.

27. Hamzeh, H. and F. Aniel, Monte Carlo study of phonon dynamics in III-V compounds. Journal of Applied Physics, 2011. 109(6): p. 063511.

28. Zebarjadi, M., A. Shakouri, and K. Esfarjani, Thermoelectric transport perpendicular to thin-film heterostructures calculated using the Monte Carlo technique. Physical Review B, 2006. 74(19): p. 195331.

29. Rowlette, J. and K.E. Goodson, Fully coupled nonequilibrium electronphonon transport in nanometer-scale silicon FETs. Electron Devices, IEEE Transactions on, 2008. 55(1): p. 220-232.

30. Sadi, T. and R.W. Kelsall, Monte Carlo study of the electrothermal phenomenon in silicon-on-insulator and silicon-germanium-on-insulator metal-oxide field-effect transistors. Journal of Applied Physics, 2010. 107(6): p. 064506.

31. Shi, Y., Z. Aksamija, and I. Knezevic, Self-consistent thermal simulation of GaAs/AlO. 45GaO. 55As quantum cascade lasers. Journal of Computational Electronics, 2012. 11(1): p. 144-151.

32. Raleva, K., et al., Modeling thermal effects in nanodevices. Electron Devices, IEEE Transactions on, 2008. 55(6): p. 1306-1316.

33. Vasileska, D., et al., Current progress in modeling self-heating effects in FD SOI devices and nanowire transistors. Journal of Computational Electronics, 2012. 11(3): p. 238-248.

34. Kamakura, Y., et al. Coupled Monte Carlo simulation of transient electronphonon transport in nanoscale devices. in Simulation of Semiconductor Processes and Devices (SISPAD), 2010 International Conference on. 2010. IEEE.

35. Ni, C., et al., Coupled electro-thermal simulation of MOSFETs. Journal of Computational Electronics, 2012. 11(1): p. 93-105.

36. Ni, C., Phonon transport models for heat conduction in sub-micron geometries with application to microelectronics, P. University, Editor. 2009.

37. Pop, E., R.W. Dutton, and K.E. Goodson, Analytic band Monte Carlo model for electron transport in Si including acoustic and optical phonon dispersion. Journal of Applied Physics, 2004. 96(9): p. 4998-5005.

38. Nghiem, T.T.T., J. Saint-Martin, and P. Dollfus, New insights into selfheating in double-gate transistors by solving Boltzmann transport equations. Journal of Applied Physics, 2014. 116(7): p. 074514.

39. Pop, E., S. Sinha, and K.E. Goodson, Heat generation and transport in nanometer-scale transistors. Proceedings of the IEEE, 2006. 94(8): p. 15871601.

40. Callaway, J., Model for lattice thermal conductivity at low temperatures. Physical Review, 1959. 113(4): p. 1046.

41. Holland, M., Analysis of lattice thermal conductivity. Physical Review, 1963. 132(6): p. 2461.

42. Glassbrenner, C. and G.A. Slack, Thermal conductivity of silicon and germanium from 3 K to the melting point. Physical Review, 1964. 134(4A): p. A1058. 
43. Debernardi, A., S. Baroni, and E. Molinari, Anharmonic phonon lifetimes in semiconductors from density-functional perturbation theory. Physical review letters, 1995. 75(9): p. 1819.

44. J. Menéndez, M.C., Temperature dependence of the first-order Raman scattering by phonons in Si, Ge, and $\alpha-S n$ : Anharmonic effects. Physical Review B, 1984. 29.

45. Ju, Y.S., Phonon heat transport in silicon nanostructures. Applied Physics Letters, 2005. 87(15): p. 153106.

46. Liu, W. and M. Asheghi, Phonon-boundary scattering in ultrathin singlecrystal silicon layers. Applied Physics Letters, 2004. 84(19): p. 3819-3821.

47. Asheghi, M., et al., Phonon-boundary scattering in thin silicon layers. Applied Physics Letters, 1997. 71(13): p. 1798-1800.

48. Liu, W. and M. Asheghi, Thermal conduction in ultrathin pure and doped single-crystal silicon layers at high temperatures. Journal of applied physics, 2005. 98(12): p. 123523.

49. Heaslet, M.A. and R.F. Warming, Radiative transport and wall temperature slip in an absorbing planar medium. International Journal of Heat and Mass Transfer, 1965. 8(7): p. 979-994.

50. Nghiêm, T.T.T., Numerical study of electro-thermal effects in silicon devices. 2013, University of Paris-Sud: Orsay, France.

51. Martin, J.S., A. Bournel, and P. Dollfus, On the ballistic transport in nanometer-scaled DG MOSFETs. Electron Devices, IEEE Transactions on, 2004. 51(7): p. 1148-1155.

52. Rhew, J.-H., Z. Ren, and M.S. Lundstrom, A numerical study of ballistic transport in a nanoscale MOSFET. Solid-State Electronics, 2002. 46(11): p. 1899-1906.

53. Rowlette, J.A. and K.E. Goodson, Fully coupled nonequilibrium electronphonon transport in nanometer-scale silicon FETs. Electron Devices, IEEE Transactions on, 2008. 55(1): p. 220-232.

54. Aubry-Fortuna, V., P. Dollfus, and S. Galdin-Retailleau, Electron effective

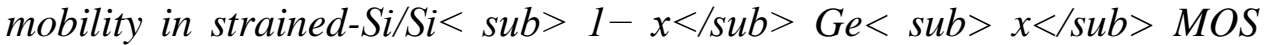
devices using Monte Carlo simulation. Solid-state electronics, 2005. 49(8): p. 1320-1329.

\section{Figure captions}

Figure 1. Thermal conductivities in bulk $\mathrm{Si}$ as a function of the temperature: experimental data [42] (triangles), model of Holland [41] (solid line), scattering parameters from [41] with the dispersion of Eq. 1 (dashed line) and our work (red crosses).

Figure 2. Thermal conductivity as a function of the roughness parameter $\Delta$ for 20 , 50, $100 \mathrm{~nm}$ and $1.6 \mu \mathrm{m}$ film thickness. Symbols are the experimental data for $\mathrm{T}_{\mathrm{Si}}=20 \mathrm{~nm}$ (triangle)[45], $50 \mathrm{~nm}$ (square)[45], $100 \mathrm{~nm}$ (diamond)[46] and $1.6 \mu \mathrm{m}$ (circle) [47]. 
Figure 3. In-plane Thermal conductivities in silicon thin-films at $300 \mathrm{~K}$ as a function of the film width. Continuous lines: our analytical RTA model, symbols: experimental data [47] (squares), [14] (blue triangle),[45] (red triangles), [46] (red circles), [48] (blue quadrangle).

Figure 4. Temperature profiles in a $5 \mu \mathrm{m}$ long Si bar: our numerical pBTE (symbols) and analytical calculation (solid line) for a large temperature gradient.

Figure 5. Effective temperature profiles for different Si bar lengths $L$ from $4 \mu \mathrm{m}$ down to $2 \mathrm{~nm}$ for a small temperature gradient.

Figure 6. Evolutions of the longitudinal wave vector $q_{z}$ spectra for TA phonons at different positions regularly distributed between the two contacts for (a) $2 \mu \mathrm{m}$ (b) $200 \mathrm{~nm}$ and (c) $20 \mathrm{~nm}$ bar lengths. The arrows show the direction from contact 1 to contact 2 .

Figure 7. Main steps of the coupled electro-thermal simulator.

Figure 8. Schematic cross-section of the simulated device.

Figure 9. At $V_{g s}=0.5 \mathrm{~V}$ and $V_{d s}=1.0 \mathrm{~V}$. (a) Heat power density extracted from eMC simulation (green solid line) and from the product (dashed red line). (b) Net phonon generation rate per mode (dashed lines) and total generation rate (continous line) due to electron-phonon scattering, from eMC simulation.

Figure 10. At $V_{g}=0.5 \mathrm{~V}$ and $V_{d s}=1.0 \mathrm{~V}$. (a) Evolution of the effective temperature $T_{\text {eff }}$ along the device for several loop numbers. (b) After convergence, profiles of $T_{\text {eff }}$ (solid lines) and $T_{\text {Fourier }}$ (dashed lines) for roughness parameters $\Delta=0.35 \mathrm{~nm}$, $1 \mathrm{~nm}$ and $3 \mathrm{~nm}$.

Figure 11. (a) $I_{d^{-}} V_{d s}$ at $V_{g}=0.5 \mathrm{~V}$ and (b) $I_{d^{-}} V_{g s}$ at $V_{d s}=1.0 \mathrm{~V}$. Open loop (blue lines with circular symbols) vs. electro-thermal simulations (red lines with triangular symbols). Roughness parameter $\Delta=3 \mathrm{~nm}$. 


\section{Table caption}

Table 3. Expressions of relaxation times for phonon-phonon interactions in Bulk Si. The values of parameters BTN, BTU and BL are discussed in the text and given in Table 2

Table 4. Set of parameters used for phonon-phonon interactions in Bulk Si when considering a quadratic dispersion. 
Figure 1.

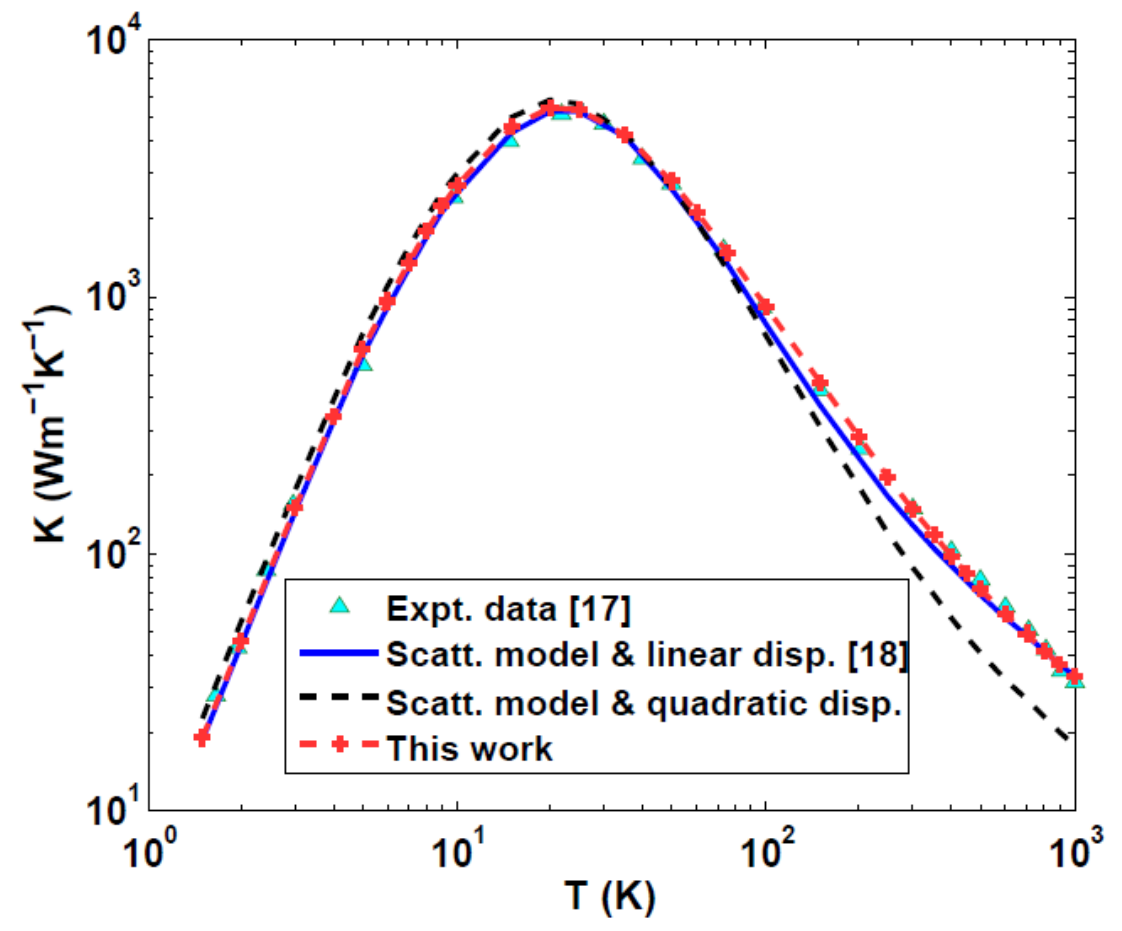


Figure 2.

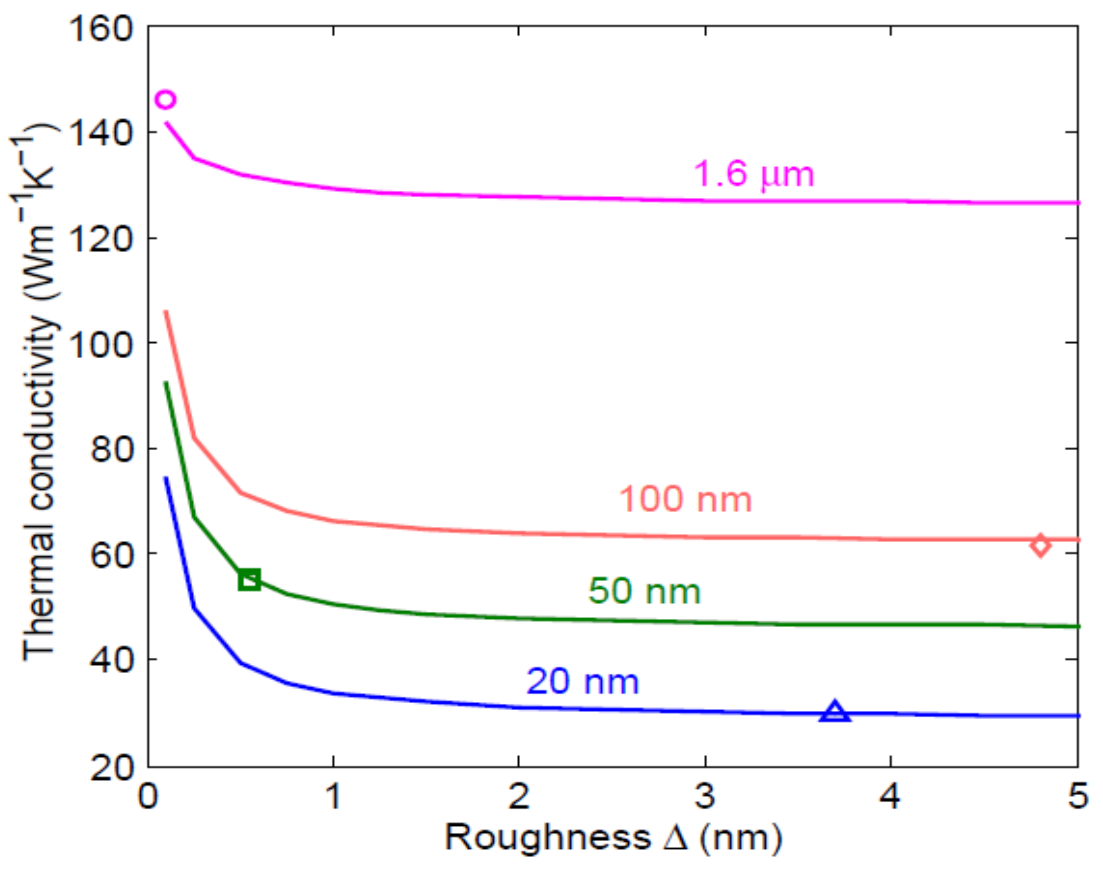


Figure 3.

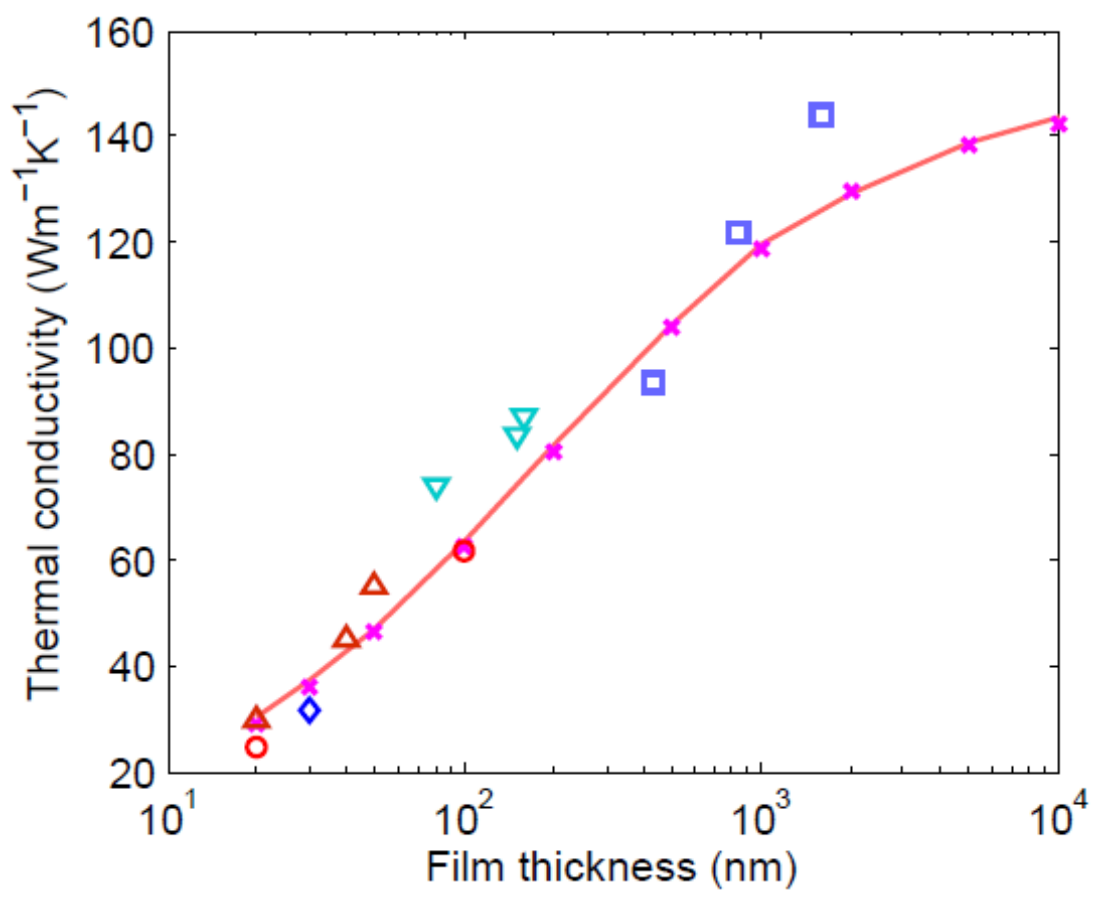


Figure 4

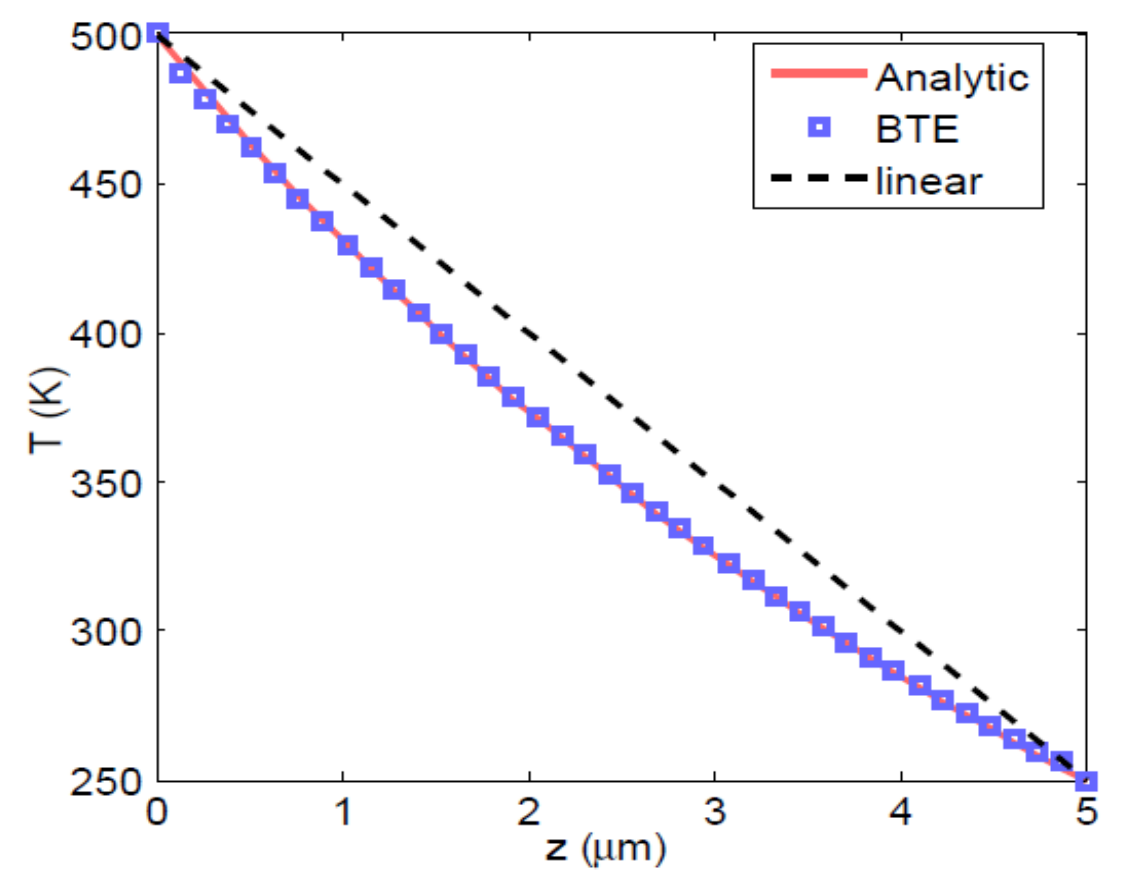


Figure 5.

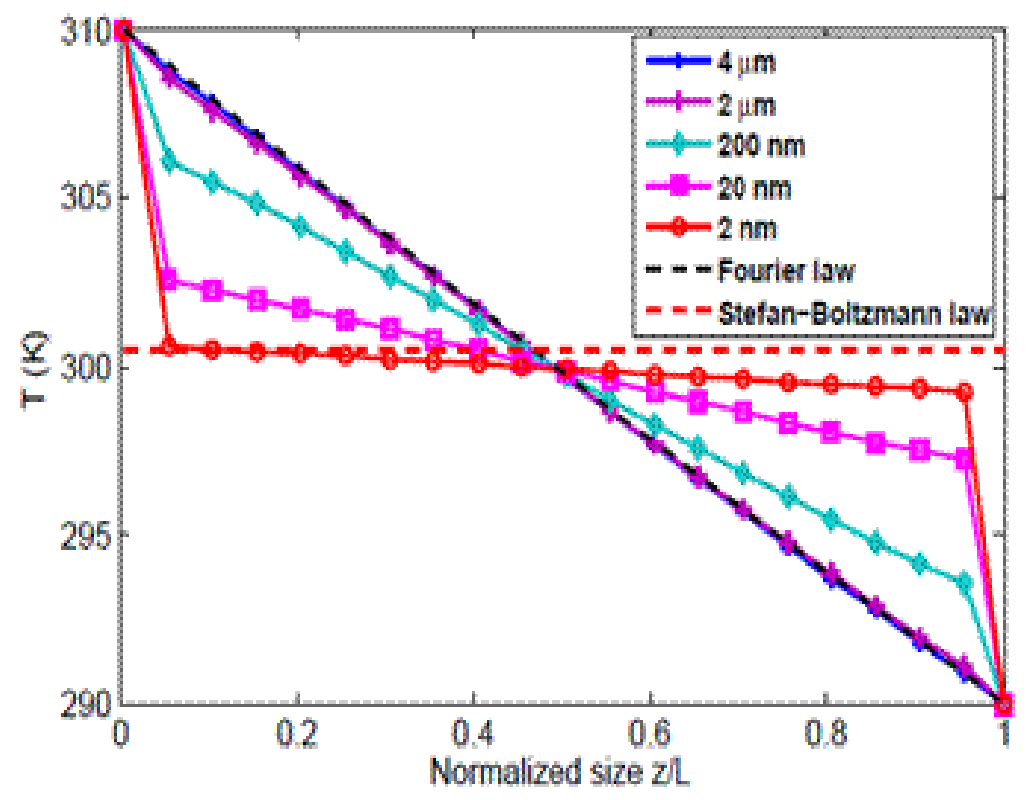


Figure 6
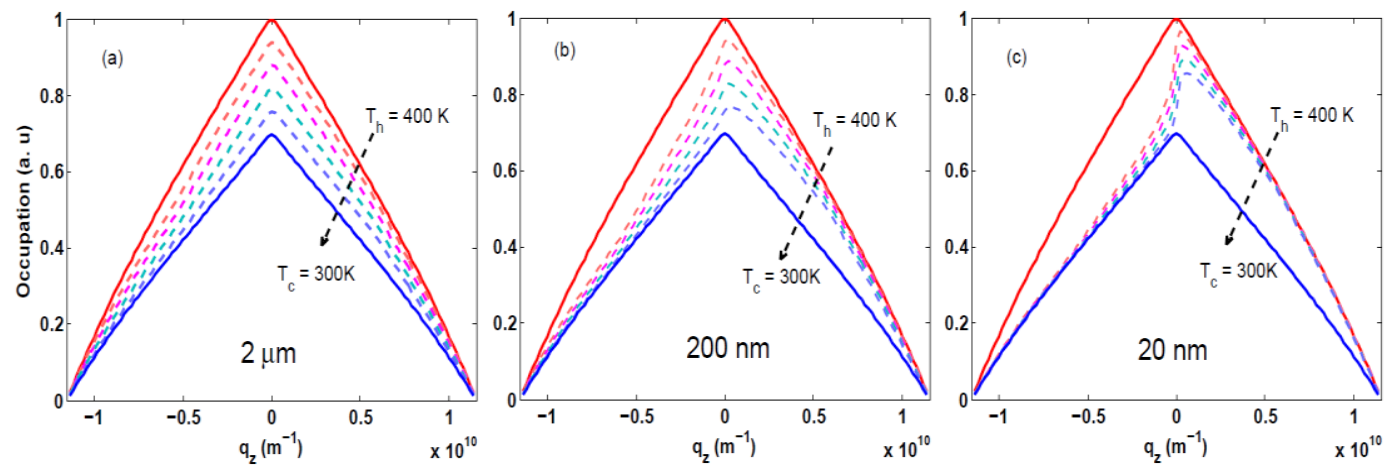
Figure 7

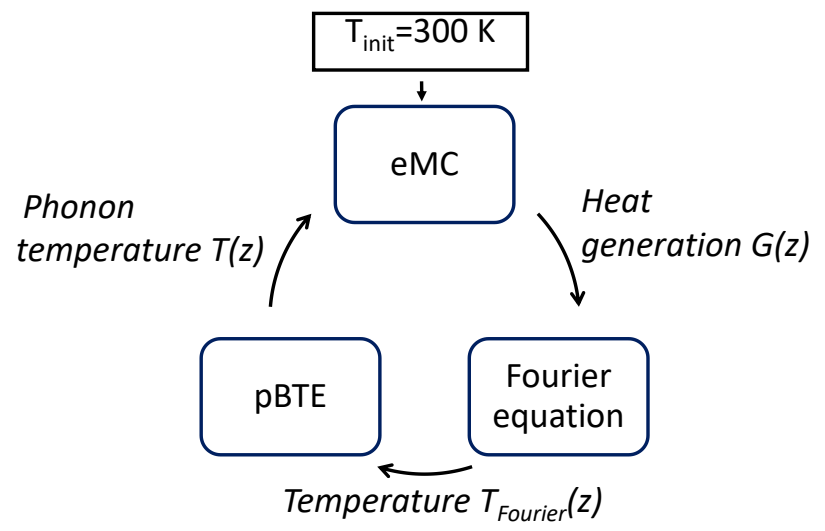


Figure 8

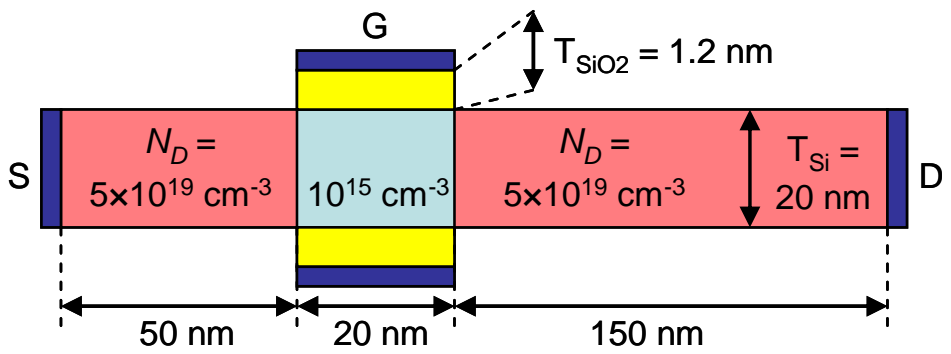


Figure 9
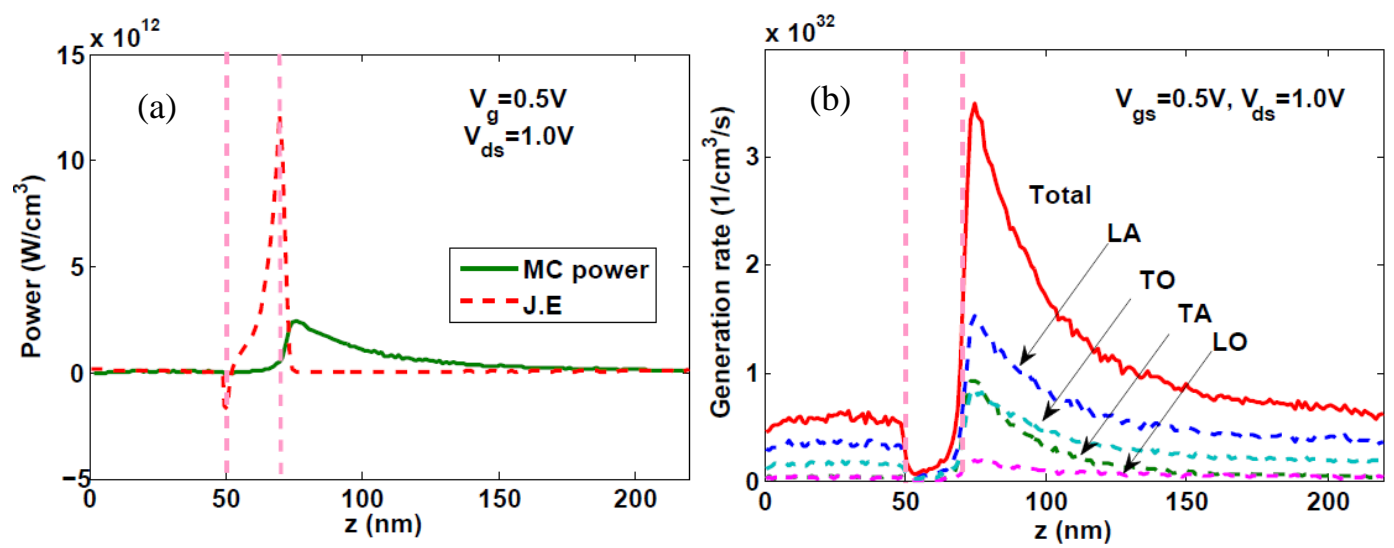
Figure 10.
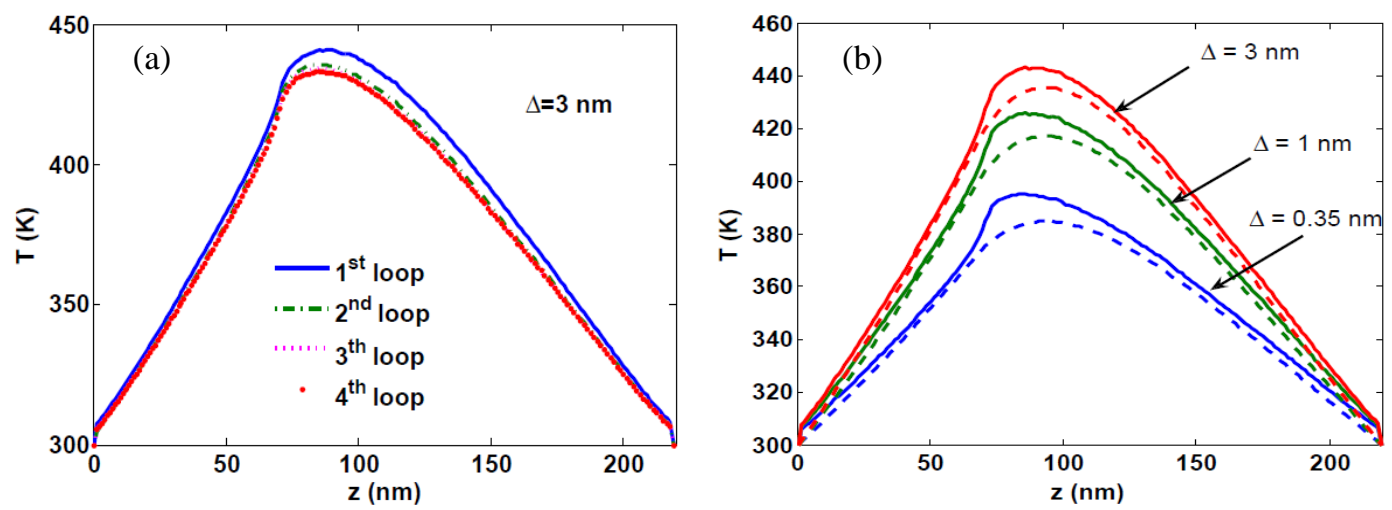
Figure 11
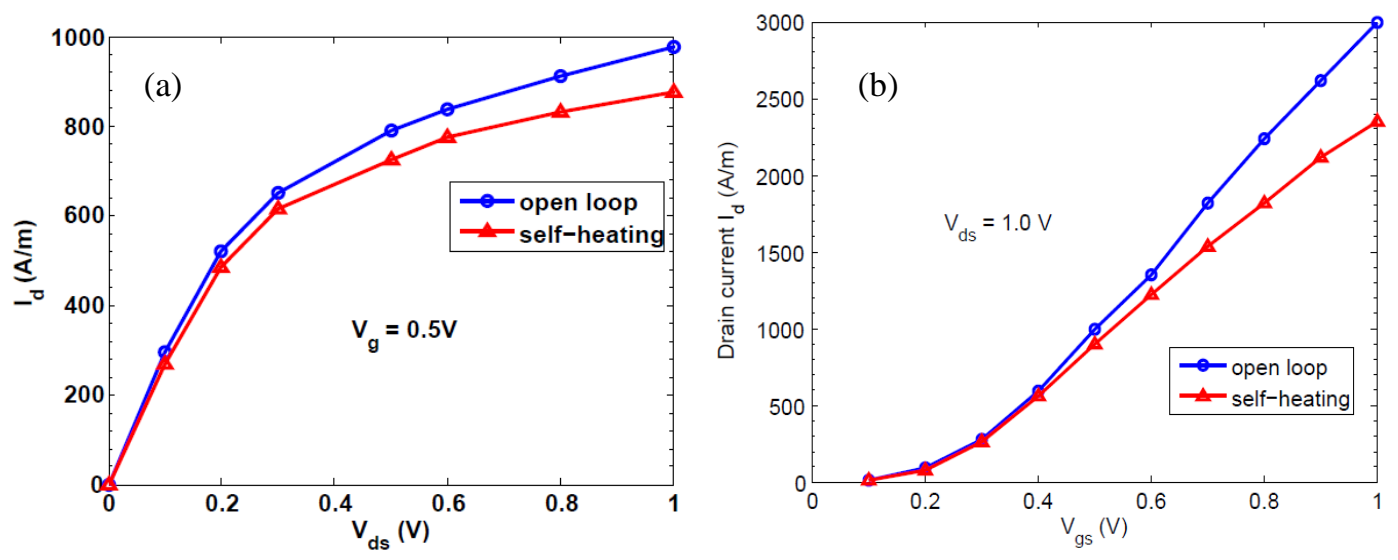